\title{
La inteligencia emocional en la educación virtual
}

\section{Emotional intelligence in virtual education}

1 María Laura Bustillos

https://orcid.org/0000-0001-5467-8739.

Universidad Técnica de Ambato, Facultad de Ciencias Humanas y de la Educación,

Carrera de Psicopedagogía, Ambato, Ecuador,

mbustillos7499@uta.edu.ec

2 Marlon Patricio Garcés Loja (iD) https://orcid.org/0000-0001-5573-7569

Universidad Técnica de Ambato, Facultad de Ciencias Humanas y de la Educación,

Carrera de Psicopedagogía, Ambato, Ecuador

mgarces2575@uta.edu.ec

3 Ángela Verónica Paredes Núñez (iD) https://orcid.org/0000-0001-6889-8695.

Universidad Uniandes, Facultad de Ciencias Administrativas, Carrera de Turismo y

Educación Básica, Ambato, Ecuador

vero_paredesn@hotmail.com

$4 \quad$ Luis Rafael Tello Vasco

https://orcid.org/0000-0003-1924-303

Universidad Técnica de Ambato, Facultad de Ciencias Humanas y de la Educación,

Carrera de Psicopedagogía, Ambato, Ecuador

ltello@uta.edu.ec

Artículo de Investigación Científica y Tecnológica

Enviado: 24/12/2021

Revisado: 29/12/2021

Aceptado: 03/01/2022

Publicado:08/03/2023

DOI: https://doi.org/10.33262/concienciadigital.v6i1.4.2041

Cítese: $\quad$ Laura Bustillos, M., Garcés Loja, M. P., Paredes Núñez, Ángela V., \& Tello Vasco, L. R. (2023). La inteligencia emocional en la educación virtual. ConcienciaDigital, 6(1.4), 931 949. https://doi.org/10.33262/concienciadigital.v6i1.4.2041

CONCIENCIA DIGITAL, es una Revista Multidisciplinar, Trimestral, que se publicará en soporte electrónico tiene como misión contribuir a la formación de profesionales competentes con visión humanística y crítica que sean capaces de exponer sus resultados investigativos y científicos en la misma medida que se promueva mediante su intervención cambios positivos en la sociedad. https://concienciadigital.org .

La revista es editada por la Editorial Ciencia Digital (Editorial de prestigio registrada en la Cámara Ecuatoriana de Libro con No de Afiliación 663) www.celibro.org.ec 


\section{Palabras} claves: inteligencia emocional, habilidades sociales, motivación, educación virtual, estrategias virtuales, modelos de enseñanza

Keywords: Emotional intelligence, social skills, Motivation, Virtual education, Virtual strategies, Teaching Models Typography
Resumen

Introducción. La inteligencia emocional es considerada como la capacidad que permite distinguir, comprender, regular y tomar conciencia de nuestras emociones, y las de los demás,tolerar las presiones y frustraciones que soportamos en el diario vivir. De tal manera que dentro de la educación la inteligencia emocional, cumple un gran papel, sobre todo en la adquisición del aprendizaje, debido a que mayor estabilidad y control emocional, mayor seráel aprendizaje significativo que se adquiera y mayor éxito se tendrá en el ámbito educativo. Es por ello por lo que la presente investigación tiene como Objetivo Determinar si la inteligencia emocional influye en la educación virtual de los estudiantes de la Facultad de Ciencias Humanas y de la Educación. Metodología: En la que se realizó con un enfoque cuantitativoaplicando el test estructurado "cuestionario inventario emocional BarOnlo" para conocerlas características de la inteligencia emocional en los estudiantes, lo cual lo transforma en un enfoque mixto, ya que esta investigación es de tipo descriptivo - exploratorio y se detalla las características marcadas de la inteligencia emocional en los estudiantes dentro del ámbito educativo y como este influye dentro del mismo. Resultados Se dio a conocer que en el componente intrapersonal es el que más control tienen los estudiantes, mientras que en componente del manejo del estrés es el que menos control presenta. Conclusión con un conjunto de herramientas virtuales y metodologías por parte del docente se puede llegar a que el estudiante aumente su capacidad de retener información por mayor tiempo.

\section{Abstract}

Introduction. Emotional intelligence is considered as the capacity that allows us to distinguish, understand, regulate, and become aware of our emotions, and those of others, to tolerate the pressures and frustrations that we endure in our daily lives. In such a way that within education, emotional intelligence plays a great role, especially in the acquisition of learning, due to the greater stability and emotional control, the greater the meaningful learning that is acquired and the greater success there will be in the educational field. That is why the present research aims to determine if emotional intelligence influences the virtual education of students of the Faculty of Human Sciences and Education. Methodology: In 
which it was carried out with a quantitative approach applying the structured test "BarOnlo emotional inventory questionnaire" to know the characteristics of emotional intelligence in students, which transforms it into a mixed approach, since this research is descriptive - Exploratory and details the marked characteristics of emotional intelligence in students withinthe educational field and how it influences within it. Results It was revealed that in the intrapersonal component it is the one with the most control by the students, while in the stressmanagement component it is the one with the least control. Conclusion with a set of virtual tools and methodologies by the teacher, the student can increase their ability to retain information for a longer time.

\section{Introducción}

\section{La inteligencia emocional}

Es considerada como una capacidad para distinguir, comprender, regular y tomar conciencia denuestras emociones, y las de los demás, tolerar las presiones y frustraciones que soportamos enel diario vivir, incrementar nuestra capacidad de empatía y nuestras habilidades sociales, permitiendo de este modo un crecimiento emocional e intelectual (Naranjo, 2019) .De tal manera que nos facilita guiar y controlar nuestro pensamiento y comportamiento ante diferentes situaciones de la vida cotidiana, además de que esta inteligencia le aporta al individuo seguridad,autonomía, autoestima alta, confianza en sí mismo, y esto le permite tener un mejor desenvolvimiento en los diferentes ámbitos, cabe destacar que la inteligencia emocional es mantener el equilibrio y saber sobrellevar los malos momentos, con una actitud positiva Salguero (2011).

Goleman en su libro titulado "Inteligencia Emocional" menciona que la capacidad de motivarse a uno mismo permite tener una estabilidad emocional plena (Goleman,2018).

Al mismo tiempo una persona que tiene desarrollada la capacidad de la inteligencia emocionaldemostrará varias de las siguientes características; les presta atención a sus emociones, conoce sus sentimientos y no los reprime, analiza sus proyectos y sueños a corto y a largo plazo, muestra un balance constante en sus acciones, no se toma nada personal, es autocritico/a con sus acciones, nota las emociones de otras personas y es empáticos, practicanla automotivación (Prieto, 2008).

Así mismo, para el desarrollo de la inteligencia emocional se debe tomar en consideración eltrato que tienen los padres con el niño durante los primeros años de vida, es decir la 
importancia que le dan, la atención que le prestan para jugar y hacerle sentir querido y aceptado por su núcleo familiar, además de la confianza que le generen sus progenitores al momento que realiza algo nuevo, esto influye mucho en la autopercepción que los niños tienende sí mismos y esto afecta en el futuro en su vida adulta, ya que si los padres dejaron vacíosemocionales en el niño durante los primeros años de vida, es muy posible que cuando sea adulto tenga dificultades para relacionarse con su entorno y busque siempre la aprobación delos demás sin importar si su bienestar emocional (Baena, 2019).

Es por ello por lo que para el desarrollo la inteligencia emocional, es necesario empezar desde tempranas edades, con la ayuda de los padres o cuidadores, quienes son los que más seguridademocional les aportan a los niños (Rangel, 2018).

Dentro de la inteligencia emocional se abarcan diferentes tipos como:

Tabla 1

Inteligencia emocional

\begin{tabular}{|c|c|}
\hline Tipos & Detalle \\
\hline Empatía & $\begin{array}{l}\text { Es la capacidad de entender cómo se sienten las } \\
\text { demás personas cuando pasanpor una situación } \\
\text { en especial (Carrillo, 2019). }\end{array}$ \\
\hline Habilidades sociales & $\begin{array}{l}\text { Es la capacidad que le permite al sujetotener } \\
\text { buenas relaciones interpersonales. Fuente } \\
\text { especificada no válida } \\
\text { (Garaigordobil, 2018). }\end{array}$ \\
\hline Autoconocimiento & $\begin{array}{l}\text { Es la capacidad de reconocer los sentimientos que } \\
\text { uno tiene y como estospuede llegar afectar en las } \\
\text { acciones que } \\
\text { realizamos (Bennett, 2019). }\end{array}$ \\
\hline Motivación & $\begin{array}{l}\text { Es la capacidad de mantenerse positivo y } \\
\text { motivado a pesar de las dificultades que se } \\
\text { presenten a la hora de realizar diferentes } \\
\text { actividades, que no salen como lo queremos } \\
\text { (McClelland, 2018). }\end{array}$ \\
\hline Autorregulación & $\begin{array}{l}\text { Es la capacidad que permite controlar nuestras } \\
\text { emociones en especial cuando influyen en } \\
\text { nuestras decisiones (López,2018). }\end{array}$ \\
\hline
\end{tabular}

Fuente: Estudio de contextos FCHE 
Dentro del ámbito educativo la inteligencia emocional es de suma importancia, debido a quepermite a los estudiantes dominar sus emociones y sentirse motivados para aprender, ademásde desarrollar habilidades sociales que son importantes a la hora del aprendizaje y la experimentación, también es necesario que los docentes deben incentivar en los estudiantes estas habilidades emocionales, afectivas y sociales, es por ello que tanto los estudiantes comolos docentes deben aprender a desarrollar estas capacidades emocionales para que exista un ambiente óptimo en el aula de clases y por ende en el proceso de enseñanza - aprendizaje y bajen las tazas de deserción académica (Extremera, 2019).

Los expertos en psicología concuerdan en que no existe un test psicométrico o prueba estandarizada que mida el grado de inteligencia emocional que una persona tiene, es por ello por lo que es difícil de categorizar. Pero más de un resultado numérico, las personas que tienen unalto grado de inteligencia emocional tienden a tener las mismas reflexiones ante las diferentessituaciones adversas (Simmons, 2018).

El cerebro racional o neo corteza que es la mente que piensa, es el encargado de hacernos consientes de la capacidad de reflexionar, analizar y meditar sobre nuestras acciones, por otrolado el cerebro emocional que es la mente que siente, es muy impulsivo y se deja llevar por las emociones que se dan en el momento y muchas de las veces es ilógico, debido a esto estasdos mentes se manejan de una manera armoniosa la mayor parte del tiempo, ya que una emoción alimenta e informa las operaciones de la mente racional y la mente racional eliminala energía de la entrada de ciertas emociones (Casafont, 2019).

\section{Desarrollo escolar}

El desarrollo escolar engloba las diferentes etapas que tiene el sujeto, este debe ser de formación y aprendizaje para obtener mejores resultados de conocimiento y a un futuro tenerexcelentes profesionales que sean de ayuda a la sociedad.

En la etapa escolar un niño desarrolla capacidades y destrezas que mejorarán el aprendizaje,por eso es importante desarrollar sus habilidades en cada ciclo académico. Para que un niñoen edad escolar pueda adquirir nuevos conocimientos se necesita de una alta inteligencia emocional porque este ayuda al estudiante a sobrellevar correctamente sus niveles estudio (Ríos, 2013).

\section{Edad escolar}

$\mathrm{Al}$ asistir a clases los niños presentan varios cambios en su personalidad, que interviene en su desarrollo emocional como las destrezas y habilidades que se consideran. El entorno escolar es importante, porque es un espacio para la socialización emocional; el profesor se convierte en un referente lleno de actitudes, comportamientos, sentimientos y emociones. Laescuela es, por excelencia la institución encargada de trasmitir conocimientos, valores, y preparar a los niños para un desempeño activo dentro de la 
sociedad. La escuela no es solo un espacio para enseñar y aprender, sino que, presenta oportunidades para la convivencia o interacción con las demás personas (Rocha, s.f.).

Factores que influyen en el desempeño escolar

Dentro de los factores que influyen en el rendimiento escolar, se encuentran los siguientes:

\section{Temperamento}

El temperamento es la manera de ser de las personas, el carácter que tiene para manejar lassituaciones cuando se presentan problemas y saber cómo solucionarlos

\section{El estado emocional}

El estado emocional se puede medir a través de test a los estudiantes para evaluar sus nivelesde ansiedad, agresividad, autoestima y depresión, obteniendo el grado de empatía que tienen. La empatía ayuda a crear un ambiente positivo en el aula de clases, fomentando actividadescomo la lectura, escritura, cálculo para la comprensión de una persona.

\section{Los padres}

Para el desempeño de los hijos en clase es necesario que los padres influyan en un procesoactivo, porque los niños se ven afectados por lo que piensen sus padres de ellos.

Para motivar a los niños a realizar actividades o tareas es necesario una motivación externaya sea dándoles premios $\mathrm{u}$ obsequios en el momento que obtienen buenos resultados o también castigándolos si fuera contario, por otro lado, algunos padres aplican una motivacióninterna que es cuando el niño se esfuerza y tiene una habilidad para conseguir resultados.

La motivación interna es muy eficaz a comparación de la externa por que los niños y en vezde verlo como algo que le dará obsequios o castigos se interesan por el aprendizaje que lo lleva a cambio. Los padres aplican una motivación interna animándolos a dar una mayor autonomía, por lo tanto, los niños prefieren elegir tareas difíciles a las fáciles mostrando curiosidad e interés por aprender cosas nuevas y agradándoles resolver problemas por su propia cuenta.

Mientras que los padres que usan un estilo permisivo se detienen y no parece interesarse porddesempeño escolar de sus hijos dando como consecuencia que estos niños obtengan puntuaciones bajas (García-Cerda et al., 2003). 


\section{Beneficios de la educación emocional}

La educación emocional es un proceso educativo, continuo y permanente, que busca desarrollar las emociones de cada ser humano, con el único fin de capacitarle para la vida y generando el bienestar personal y social.

Tiene varios beneficios que son los siguientes:Promueve el bienestar personal.

- Alcanza un éxito académico.

- Reduce problemas sociales y culturales relacionados con la roga, alcohol, violencia,discriminación.

○ Mejora la convivencia en el aula.

- Favorecer la salud mental y el desarrollo integral a largo o corto plazo.

\section{Educación emocional}

Es un proceso pedagógico en el que se desarrolla y entrena competencias que engloba a la inteligencia emocional; debe cumplir con varias características como:1) ser estructurado y sistematizado, 2) ser vivencial capaz de generar emociones y aprendizajes, 3) perdurar en eltiempo, 4) generar hábitos en la etapa escolar.

En las instituciones educativas cada vez son más que incluyen su proyecto educativo con objetivos, metodología, y actividades basadas en la educación emocional para el desarrollo de la inteligencia emocional.

\section{Desarrollo cognitivo}

En el momento de nacer hasta la etapa adulta, pasamos por un proceso de crecimiento y a lolargo de la vida se produce un crecimiento físico y un desarrollo psicológico. Existen diferentes etapas evolutivas del desarrollo psicológico por las que pasan las personas, cada una con sus características, cada momento está definido con variaciones individuales que debemos conocer para educar a los más pequeños.

\section{Educación virtual}

La educación en línea o educación virtual es un concepto al desarrollo de programas de formación con un escenario de enseñanza y aprendizaje, establece un dialogo o experiencia de aprendizaje sin darse un encuentro profesor y alumno de manera presencial y con la capacidad de establecer una relación interpersonal y de carácter educativo.

Es una acción que busca espacios de formación apoyándose en las TIC y lograr la enseñanzay aprendizaje de manera novedosa; es una modalidad de la educación a distancia que implicaexigencias del entorno político, económico y social, relaciones pedagógicas y las TIC. 
La educación a distancia de manera virtual tiene sus inicios por la radiodifusión y se desarrolló hasta ofrecer cursos sostenidos en lo audiovisual a través de las vías satelitales, refiriéndose a la enseñanza a distancia caracterizada por el alumno, profesor y el contenido. El material didáctico se convierte en un elemento relevante que incluye y ofrece informaciónal alumno donde interviene el autoaprendizaje (Bossis et al., 2015).

El tutor puede contemplar las modalidades combinadas con metodologías y contenidos usados proporcionando acompañamiento orientado a los alumnos en su aprendizaje, resolviendo dudas de los temas usando pautas y estrategias dependiendo de cursos, en este sistema se utiliza medios tecnológicos, aprendizajes independientes y una tutoría cada cierto bmp estos elementos definen al proceso educativo con la formación y la comunicación delos autores en el sistema educativo.

La tecnología es una herramienta fundamental para una educación con avances y eficacia. Sedebe aprovechar a la tecnología para comprender los contextos educativos, con un apoyo al proceso de enseñanza y aprendizaje en todos los niveles educativos formales y no formales.Los seres humanos tienen la capacidad de tomar acciones sistemáticas, perfeccionarlos y enseñarlos sin importar la distancia y el tiempo; toman decisiones según convengan, el ser humano no solo se caracteriza por elaborar aparatos o herramientas sino también por la capacidad de usarlos y crear tecnologías, sistemas de pensamientos y lenguajes (Aldana et al., 2016).

En la educación virtual los docentes y estudiantes interactúan de manera indirecta o sin estarfrente a frente, este proceso es modificable frente a la enseñanza y aprendizaje, mejorando lametodología con la integración de disciplinas de estudio y sus contenidos, esto revoluciona al sistema educativo para nuestro país permitiendo una nueva forma de educar siendo solidarios, honestos, equitativos, con un desarrollo cultural en Ecuador.

\section{Estrategias virtuales}

- El mapeo establece metas a corto, mediano y largo plazo donde se recolecta la información, con el mapeo se obtiene datos relevantes del estudiante como son las fortalezas, debilidades, gustos, sueños y temores, comunicación del estudiante y sus rutinas diarias.

- Plan Centrado en la Persona (PCP) establece contenidos académicos alineados al currículo nacional complementarios a la comunicación, habilidades motoras, orientación, de acuerdo con las necesidades, identificando apoyos al estudiante para alcanzar su aprendizaje.

- Videos tutoriales personalizados fue implementada para cumplir el proceso de enseñanza especificando destrezas trabajadas con el estudiante.

- Clase virtual individual donde se explica la actividad a realizar adecuando un horario prudencial respetando los horarios del estudiante para una mejor comprensión del 
contenido.

- Refuerzo académico el objetivo es dar seguimiento, acompañamiento y retroalimentación, se puede dar mediante videos tutoriales, permitiendo saber si los contenidos fueron aprendidos para ser aplicados en los contextos.

Modelos de enseñanza/aprendizaje en la educación

Modelo presencial tradicional: Este método es tradicional caracterizando a los estudiantesde forma física donde se comparte el tiempo y espacio recibiendo sus clases a través de la comunicación oral

Modelo de educación a distancia: Se caracteriza por la no presencialidad y se utilizan medios de distribución de información con la utilización de productos electrónicos y tecnológicos para la distribución de la información.

Modelos de enseñanza/aprendizaje virtual e-learning: Usa soportes tecnológicos para la información y comunicación como los cursos virtuales "online", chats entre profesores y alumnos, mensajes instantáneos mediante redes sociales comunes, video llamadas, videoconferencias, información en la red.

Modelos de enseñanza/aprendizaje virtual mixto: "Blended learning", consiste en una modalidad semipresencial que incluye información virtual y formación presencial

\section{Modelo de enseñanza virtual}

La interacción entre profesor y alumno entre si es una nueva forma educacional mediante latecnología con diferentes características dentro del contexto tradicional o presencial.

El modelo de enseñanza virtual, el alumnado pasa a ser protagonista y el profesorado mediante distintas funciones como son los contenidos virtuales, trabajos, proyectos, enlaces a páginas web, referencias bibliográficas online y autoevaluaciones proporcionando guías yorientaciones.

\section{Manejo de las emociones en la educación virtual}

El aprendizaje virtual con las emociones se relaciona entre sí, últimas investigaciones demuestran que el aprendizaje mejora en el momento que disminuye las emociones negativasy aumenta sus emociones positivas.

La emoción es un estado afectivo en la que se siente como algo placentero o desagradable según sea su reacción; la neurociencia contribuye a comprender la relación entre la enseñanzay aprendizaje con las emociones. Toda información que ingresa por medio de nuestros sentidos es procesada por el cerebro para luego ser racionalizada (Avedaño, 2020). 
Los estudiantes pueden percibir emociones positivas o negativas como son:

Frustración: los estudiantes sienten esto en el momento que una instrucción no es clara, deficiente o poco relevante de la información, todo esto lleva a que sea un obstáculo. También puede ser cuando el tutor lo aíslan o no son considerados en una retroalimentación.

Ansiedad: Aparece por la falta de experiencia con la tecnología. La necesidad de comunicación y participación constante, los horarios, fechas y tiempo límite para realizar alguna actividad (Berrocal, 2019).

Vergüenza: Un estudiante pude sentirse así, en el momento que no logra completar una tareay se pone en manifiesto hacia los demás cuando expone su trabajo hacia personas desconocidas, cuando una persona se siente avergonzada su aprendizaje disminuye notablemente.

Entusiasmo: Se asocia a la emoción de experimentar algo nuevo o una forma de aprender diferente como por ejemplo el uso de nuevas tecnologías, la posibilidad de conectarse con otros profesionales.

Orgullo: El estudiante experimenta el orgullo al tener éxito al completar un trabajo o proyecto, reconocimiento o retroalimentación. Las emociones en el aprendizaje virtual son relevantes. El orgullo es importante para alimentar la motivación del estudiante y crea una mejor disposición.

Las herramientas utilizadas y el afecto positivo conducen a un mayor conocimiento, eficacia y experiencia, y un afecto negativo conducirá a evitar una herramienta de aprendizaje (Echeverri, 2020).

En la última década, las concepciones científicas abren camino para una investigación científica- educativa, las teorías sociales han supuesto referentes novedosos que hablan sobrenuevas concepciones respecto a las personas y la educación. La relación emocional con los contenidos y las herramientas supone interés con el e-Learning y teleformaciones, especialmente que la calidad de la educación se evalúa y mide a través de varios indicadores etelos que se visualiza el abandono de los estudios y se sintetizan con factores asociados como la baja autoestima, falta de recompensas o superación de obstáculos, escasez de integración social y académica. Otros estudios revelan sobre el fracaso escolar en las que secaracteriza por la cultura escolar y la regulación de emociones (Catalán, 2018).

En la actualidad los estudiantes sienten síntomas y trastornos relacionados con la ansiedad, depresión, e ira, que se caracteriza por el agotamiento, baja realización personal. Estos problemas de salud mental se agravan con alteraciones fisiológicas como 
consecuencia de diversos estresores. Se analizado la relación entre la inteligencia emocional con la actividad delestudiante y su ámbito escolar, por lo que se dio más importancia al fortalecimiento de la inteligencia emocional (Chiappe, 2017).

\section{El aprendizaje en la educación virtual:}

La educación virtual o también conocida como enseñanza en línea, hace referencia al desarrollo de la dinámica de enseñanza - aprendizaje que es realizado de forma virtual. Es decir, existe un formato educativo en donde los docentes y estudiantes pueden interactuar diferente al espacio presencial.

Esta modalidad de educación se basa en el uso TIC's o las nuevas tecnologías, ya que hace uso de las herramientas virtuales que a través del internet proporcionan ambientes educativosadecuados y de alta calidad. Pero es importante tomar en cuenta que la educación virtual se relaciona con la educación a distancia, la cual nació a raíz de la necesidad de cobertura de calidad educativa a personas que, por distancia y tiempo, no pueden desplazarse hacia un centro de formación de manera física (Bhat et al., 2010).

La educación virtual complementa a este tipo de modalidad académica, ya que mejora las dinámicas de formación de los estudiantes y les ofrece mayor apoyo a los docentes para el desarrollo y seguimiento académico de los alumnos a cargo. Veamos a continuación, la evolución que ha tenido la educación a distancia y su relación con la educación virtual.

\section{La influencia de las emociones en la educación virtual:}

Las emociones son de suma importancia en los procesos cognitivos, ya que motiva a los estudiantes a tomar riesgos y experimentar diferentes posibilidades, para encontrar soluciones adecuadas. Es por ello por lo que los estudiantes rinden de mejor manera al aumentar sumotivación y disminuir el estrés y la ansiedad. Del mismo modo que si el estudiante se sientemotivado y se mantiene estable emocionalmente, el aprendizaje fluye de manera positiva y llega a ser significativo, por lo contrario, si el estudiante tiene una inestabilidad emocional y se somete a altos grados de estrés no podrá adquirir el aprendizaje (Tartaj et al., 2005).

Además, la neurociencia nos ha permitido entender la relación que existe entre las emociones y el proceso de enseñanza - aprendizaje, ya que toda la información que percibimos a través de nuestros sentidos, son procesados emocionalmente antes que racionalmente. Dentro del aprendizaje virtual los estudiantes suelen sentirse aburridos y desmotivados, frustrados, presentan cuadros de ansiedad y depresión, además de que se cohíben de hablar y participaren clases, y esto imposibilita que el proceso de enseñanza - aprendizaje se dé de la manera correcta. 
Herramientas digitales que se hacen uso en la educación virtual:

Las herramientas digitales o virtuales usadas para la educación son varios programas que engloban diferentes tipos de herramientas destinadas a ayudar a los docentes al momento deimpartir las clases. Su principal función es facilitar la creación de entornos virtuales para impartir todo tipo de información de manera dinámica y diferente y llamar la atención de los estudiantes, a través de internet sin necesidad de tener conocimientos de programación. Además, estas herramientas nos sirven para cualquier tipo de asignatura o tema a tratar, es porello por lo que facilita el trabajo del docente y el aprendizaje de los estudiantes (Casares, 2020).

En la actualidad, la tecnología es un modificador que permite hacer de la educación un proceso más divertido y fácil, es por ello por lo que de la tecnología se derivan las herramientas digitales que benefician el trabajo de los docentes y de los estudiantes a la hora del aprendizaje. Entre las herramientas que se pueden hacer uso tenemos las siguientes; Canva, Educaplay, Quizzes, Kahoot, Knovio, Filmora Go, Recap, Zoom, Edmodo, Blogger, Google Drive, Office 365, entre otros, que son fáciles de utilizar y permiten hacer de la clase más dinámicay diferentes.

\section{Metodología}

El presente trabajo investigativo se realizó en la Universidad Técnica de Ambato a 103 estudiantes de la carrera de Psicopedagogía, en la que se realizó con un enfoque cuantitativo, aplicando un test estructurado "Cuestionario Inventario emocional BarOnlo", para dar resultados exactos y cualitativos ya que se prestan para dar opiniones e interpretaciones acercade los distintas características de la inteligencia emocional en los estudiantes en la modalidadvirtual que se revisó en la investigación, lo cual lo transforma en un enfoque mixto para la investigación, además esta investigación es de tipo descriptivo - exploratorio ya que se detallalas características marcadas de la inteligencia emocional en los estudiantes dentro del ámbitoeducativo y como este influye dentro del mismo. . En los resultados más relevantes se dio a conocer que en el componente intrapersonal es el que más control tienen los estudiantes, mientras que en componente del manejo del estrés es el que menos controlpresenta.

\section{Resultados}

En la aplicación del "Cuestionario Inventario emocional BarOnlo". Ha dado como resultado 5 graficas de los componentes: interpersonal, adaptabilidad, intrapersonal, manejo del estrésy estado del ánimo en general, respectivamente.

En el componente interpersonal el 25,94\% de los encuestados tienen un nivel bajo de empatía, por otro lado, el 30,50\% tiene un nivel medio de responsabilidad social y del mismo modo el $33,83 \%$ tienen un nivel alto de relación interpersonal dando un total de 
90,27

Tabla 1

Componente Interpersonal

\begin{tabular}{|l|c|c|c|c|}
\hline \multicolumn{1}{|c|}{} & $\begin{array}{c}\text { EM } \\
\text { (empatía) }\end{array}$ & $\begin{array}{c}\text { RI } \\
\text { (relación interpersonal) }\end{array}$ & $\begin{array}{c}\text { RS } \\
\text { (responsabilidad social) }\end{array}$ & Total \\
\hline Cantidad & 25,94 & 33,83 & 30,50 & 90,27 \\
\hline
\end{tabular}

Fuente: Estudio de contextos FCHE

En el componente adaptabilidad el 25,11\% de los encuestados tienen un nivel bajo de solución de problemas, por otro lado, el $21,12 \%$ tiene un nivel medio de flexibilidady del mismo modo el $27,18 \%$ tienen un nivel alto de prueba de realidad dando un total de 73,41 .

Tabla 2

Componente de adaptabilidad

\begin{tabular}{|l|c|c|c|c|}
\cline { 3 - 5 } & $\begin{array}{c}\text { SP (solución de } \\
\text { problemas) }\end{array}$ & $\begin{array}{c}\text { PR (prueba de } \\
\text { realidad) }\end{array}$ & FL (flexibilidad) & Total \\
\hline Cantidad 25,11 & 27,18 & 21,12 & 73,41 \\
\hline
\end{tabular}

Fuente: Estudio de contextos FCHE

En el componente intrapersonal, los encuestados tienen un nivel de independencia del $15,89 \%$, por otro lado, en asertividad tiene el 18,97\%, además en comprensión emocional presentan un $24,57 \%$, y del mismo modo mostro un nivel de la autorrealización con un $26,35 \%$ y un nivel de auto concepto con el 27,46\% dando untotal de 113,24.

Tabla 3

Componente Intrapersonal

\begin{tabular}{|c|c|c|c|c|c|c|}
\hline & $\begin{array}{c}\mathrm{CM} \\
\text { (comprensión } \\
\text { emocional) }\end{array}$ & $\begin{array}{c}\text { AS } \\
\text { (asertividad) }\end{array}$ & AC (autoconcepto) & $\begin{array}{c}\mathrm{AR} \\
\text { (autorealizacion) }\end{array}$ & $\begin{array}{c}\text { IN } \\
\text { (independencia) }\end{array}$ & Total \\
\hline Cantidad & 24,57 & 18,97 & 27,46 & 26,35 & 15,89 & 113,24 \\
\hline
\end{tabular}

Fuente: Estudio de contextos FCHE

En el componente manejo del estrés, los encuestados tienen un nivel de control de impulsos del $21,85 \%$ por lo que necesita mejorar y tiene una capacidad emocional extremadamente por debajo del promedio y un nivel de tolerancia al estrés del 26,21\% es decir que necesita mejorar y tiene una capacidad emocional por debajo delpromedio, dando un total de 48,07 . 


\section{Tabla 4}

Componente del estrés

\begin{tabular}{|c|c|c|c|}
\hline & TE (tolerancia al estrés) & $\begin{array}{l}\text { CI (control de } \\
\text { impulsos) }\end{array}$ & Total \\
\hline Cantidad & 26,21 & 21,85 & 48,07 \\
\hline
\end{tabular}

Fuente: Estudio de contextos FCHE

En el componente estado del ánimo en general, los encuestados tienen un nivel en control del $26,33 \%$ y el nivel de felicidad del $26,86 \%$, en ambos subcomponentes se considera que se necesita mejorar ya que la capacidad emocional está extremadamentepor debajo del promedio dando un total de 53,19. Finalizando que los diferentes factores que se encuentra sometidos los individuos si influyen en el proceso de enseñanza y aprendizaje

\section{Tabla 5}

Componente Estado de animo en general

\begin{tabular}{|c|c|c|c|}
\hline & $\begin{array}{c}\text { FE } \\
\text { (felicidad }\end{array}$ & OP (optimismo) & Total \\
\hline Cantidad & 26,86 & 26,33 & 53,19 \\
\hline
\end{tabular}

Fuente: Estudio de contextos FCHE

La correlación entre el cuestionario BarOnlo, y la educación virtual es de 0,890, lo cual reflejaque los estudiantes de la carrera de psicopedagogía de la Universidad Técnica de Ambato, tienenuna gran relación en el componente intrapersonal con la educación virtual por varios factores que influyen en ellos como son: la comprensión, asertividad, autoconcepto, independencia, y junto a estrategias y aplicaciones virtuales por parte de los docentes haciendo que sea más llevadero en el momento de aprender algo nuevo dentro del aula de clases. 
Figura 1

Estudio de contexto

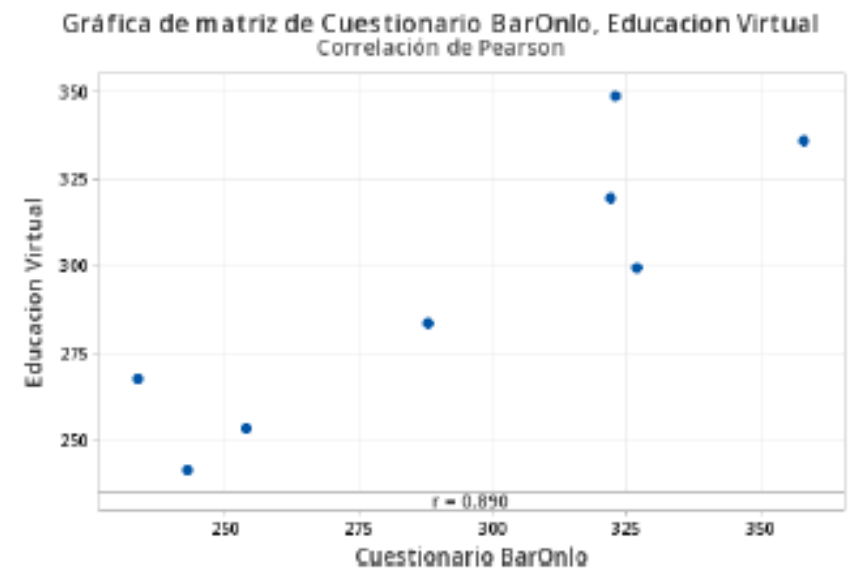

Fuente: Estudio de contextos FCHE

En el cuestionario inventario Baronlo que se realizó a estudiantes de psicopedagogía dio como resultado que el $1 \%$ se deja llevar por sus emociones positivas o negativas para realizaruna determinada acción, el 38\% tiene la capacidad de expresar sus sentimientos, emocionesy opiniones con el fin que se distinga de los demás, el $42 \%$ sabe lo que tiene y lo que quierecon respecto al autoestima, ya que si el autoestima tiene elevado será más fácil que aprenda algo y mientras tenga un autoestima baja, le será dificultoso aprender, el $17 \%$ piensa que mientras mejor hablen de uno mismo será le caerá bien a los demás, y el $5 \%$ cree que debe hacerse valer por si mismo sin necesidad de ayuda aparte.

\section{Figura 2}

\section{Emociones}

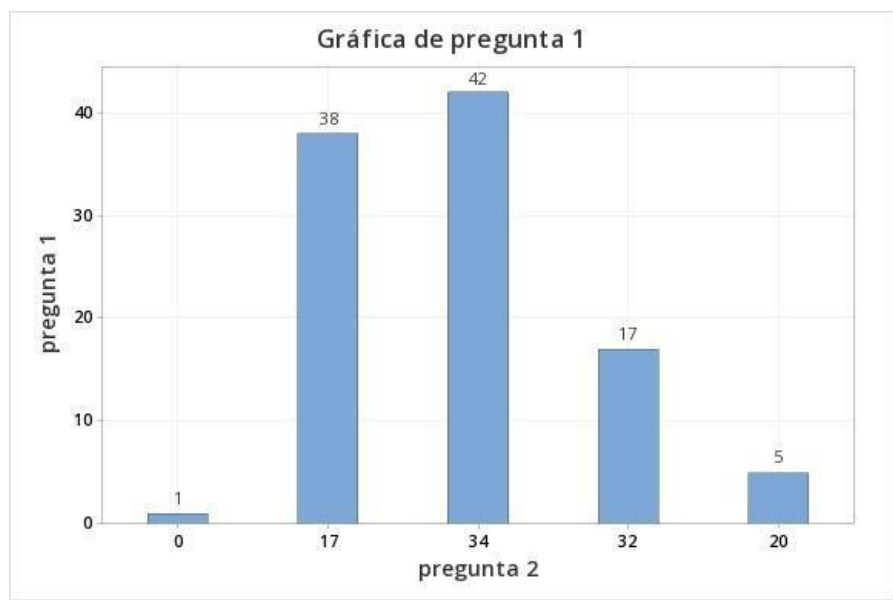

Fuente: Estudio de contextos FCHE

En el cuestionario de educación virtual que se realizó a estudiantes de psicopedagogíadio como resultado que el $38 \%$ siempre le cuesta concentrarse en aula de clases, el $43 \%$ casi 
siempre se siente motivado para participar en clase, el $17 \%$ considera que ha tenido un aprendizaje significativo en el aula de clases y el 5\% cuenta con un buen servicio de internet,dando como resultado que las facilidades que tiene cada estudiante se aumenta las ganas de aprender

\section{Figura 3}

Educación Virtual

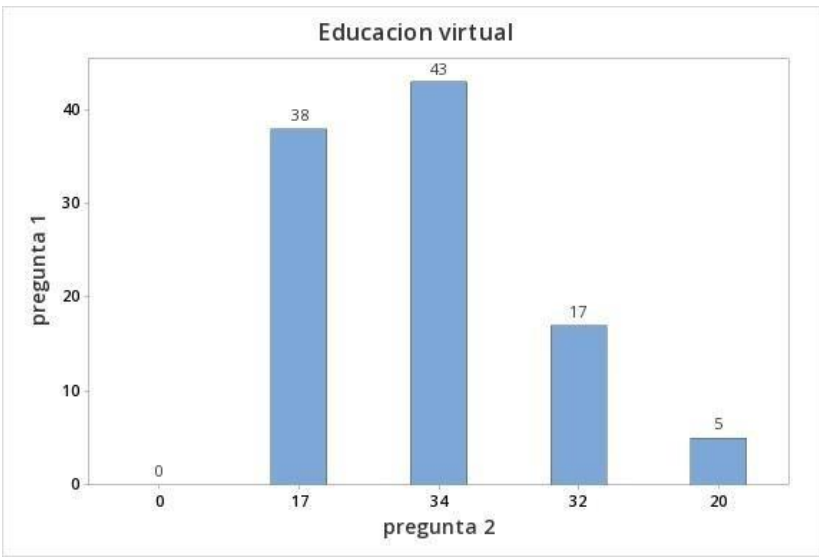

Fuente: Estudio de contextos FCHE

\section{Conclusiones}

- La inteligencia emocional es una de las capacidades del ser humano ante las situaciones de la vida, la persona es apta de controlar sus emociones para la toma dedecisiones acertadas; más aún para el sector educativo en la que el estudiante debe aprender a sobrellevar sus emociones ante los problemas que se pueda presentar en sus clases en línea. Es decir, existen varios factores que influyen en el desempeño escolar como el temperamento, padres y estado emocional presente en cada uno de ellos.

- En la presente investigación se determinó que la inteligencia emocional influye significativamente en la educación virtual debido al test realizado a los jóvenes universitarios, los resultados arrojados afirman que el componente intrapersonal es elque más control tienen los estudiantes, mientras que el estrés es el que menos controlpresentan.

- El proceso de aprendizaje resulta bajo cuando el estado de ánimo de los estudiantes presenta cuadro de depresión o bajos estados de ánimo, es decir, que el alumno no se concentra en sus clases, provocando niveles de estrés que muchas de las veces nolas pueden controlar causando algunas enfermedades.

- Las herramientas virtuales y metodologías impartidas por el docente son necesarias para que el estudiante aumente su capacidad de retención de la información por unmayor tiempo, siendo de ayuda a su capacitación. 


\section{Referencias Bibliográficas}

Aldana, S., Vereda, F., Hidalgo-Álvarez, R., \& de Vicente, J. (2016). Facile synthesis of magnetic agarose microfibers by directed self-assembly. Polymer 93, 61-64.

Avedaño, W. (2020). Educación virtual en tiempos de COVID-19: percepciones de estudiantesuniversitarios. Formación Universitaria, 119-128.

Baena, G. P. (2019). Cómo desarrollar la inteligencia emocional infantil. Guía para padres y maestros,2- 4.

Bennett, M. (2019). Autoconocimiento. Ediciones

Berrocal, P. (2019). La importancia de la inteligencia emocional. La Inteligencia Emocional 2.

Bhat, S., Tripathi, A., \& Kumar, A. (2010). Supermacroprous chitosan - agarose - gelatin cryogels in vitro characterization and in vivo assessment for cartilage tissue engineering. Journal of the Royal Society Interface, 1-15.

Bossis, G., Marins, J., Kuzhir, P., Volkova, O., \& Zubarev, A. (2015). Functionalized microfibers for field-responsive materials and biological applications. Journal of Intelligent Material Systemsand Structures, 1-9.

Carrillo, O. (2019). Evolución conceptual de la Empatía. Iniciación a la Investigación, 4.

Casafont, R. (2019). Viaje a tu cerebro emocional. Una inmersión en el mundo de las emociones., 1-3.Casares, A. (2020). Herramientas Digitales. Digitalización de la Educación, 7-8.

Catalán, R. (2018). Las emociones en el aprendizaje online. Relieve, 12-20.

Chiappe, A. (2017). Fortalecimiento de las habilidades emocionales de los educadores. Educación y educadores, 5-19.

Echeverri, A. (2020). Inteligencia emocional en educación virtual a distancia en los estudiantes deeducación primaria de la RED Campi-Huayqui Cusco. 10-23.

Extremera, N. (2019). LA IMPORTANCIA DE DESARROLLAR LA INTELIGENCIA EMOCIONAL. Inteligencia Emocional, 1.

Garaigordobil, M. (2018). Intervención en las habilidades sociales. Efectos en la inteligencia emocionaly la conducta social, 22. 
García-Cerda, L., Rodríguez-Fernández, O., Betancourt-Galindo, R., Saldívar-Guerrero, R., \& Torres-Torres, M. (2003). Síntesis y propiedades de ferrofluidos de magnetita. Superficies y Vacío,28-31.

Goleman, D. (2018). Inteligencia Emocional. Best Seller Mundial.

López, M. (2018). La autorregulación emocional como elemento central de la inteligencia emocional. Psicología Online, 6-7.

McClelland, C. D. (2018). Estudio de la motivación humana. Narcea Ediciones.Naranjo, R. A. (2019). Inteligencia Emocional Wiki. Academia, 1-2.

Prieto, M. D. (2008). Inteligencia emocional y alta habilidad. Revista española de pedagogía, 241-259.

Rangel, A. (2018). Inteligencia emocional. Salud y Vida, 1.

Ríos, B. M. (2013). Asesoría académica: un recurso para los estudiantes. Investigación en medicinaeducativa.

Rocha, M. (s.f.). La tutoría, una alternativa para contribuir a disminuir el fracaso escolar en elbachillerato. Investigación en Educación.

Salguero, M. J. (2011). Importancia de la Inteligencia Emocional. Dialnet, 2-3.Simmons, S. (2018). Cómo medir la inteligencia emocional (Vol. 16). Edaf.

Tartaj, P., Morales, M., González-Carreño, T., Veintemillas-Verdaguer, S., \& Serna, C. (2005). Advances in magnetic nanoparticles for biotechnology applications. Journal of Magnetism andMagnetic Materials, 290, 28-34.

\section{Liencia}


El artículo que se publica es de exclusiva responsabilidad de los autores y no necesariamente reflejan el pensamiento de la Revista Conciencia Digital.

\section{Ciencia \\ Ligital}

El artículo queda en propiedad de la revista y, por tanto, su publicación parcial y/o total en otro medio tiene que ser autorizado por el director de la Revista Conciencia Digital.
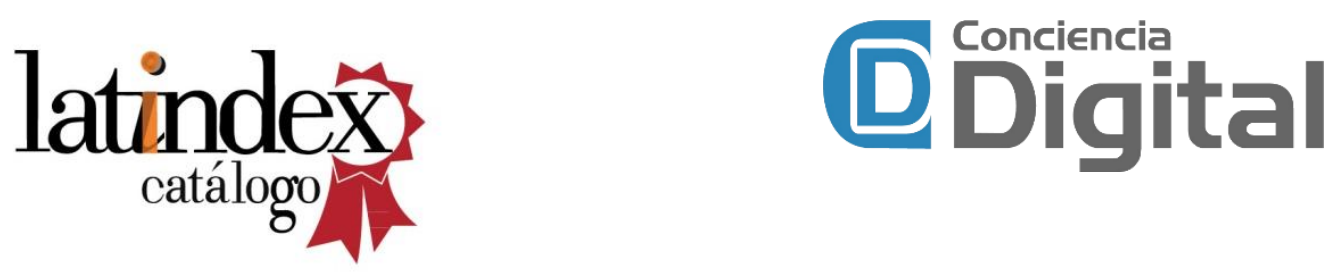

Indexaciones

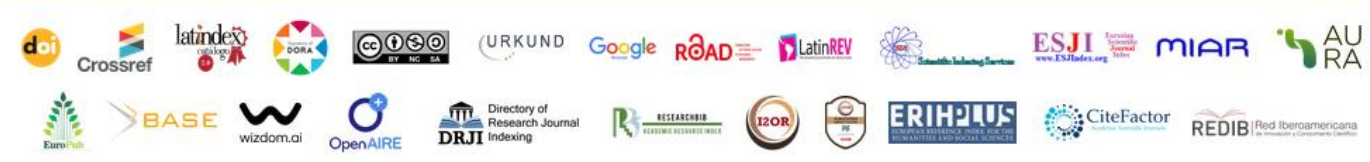

